\title{
Screening for Syphilis Infection in Pregnant Women: Evidence for the U.S. Preventive Services Task Force Reaffirmation Recommendation Statement
}

Tracy Wolff, MD, MPH; Erica Shelton, MD, MPH; Cecili Sessions, MD, MPH; and Therese Miller, DrPH

Background: In 2004, the U.S. Preventive Services Task Force strongly recommended that clinicians screen all pregnant women for syphilis infection.

Purpose: To update the evidence on screening pregnant women for syphilis infection.

Data Sources: MEDLINE searches from 1 January 2003 through 31 July 2008, recent systematic reviews, reference lists of retrieved articles, and expert suggestions.

Study Selection: English-language studies were selected to answer the following 2 questions: Does screening for syphilis in pregnancy reduce the prevalence of congenital syphilis in neonates? Are there harms of screening for syphilis or harms of treatment with penicillin in pregnancy to women or neonates? Randomized, controlled trials; meta-analyses; systematic reviews; cohort studies; and ecologic studies were selected for the potential benefits question. Randomized, controlled trials; meta-analyses; systematic reviews; cohort studies; case-control studies; and large case series were selected for the potential harms question.

Data Extraction: Information on the study design, selection criteria, demographic characteristics, and clinical outcomes was extracted from each study.
Data Synthesis: One study on benefits evaluated the effect before and after the implementation of a universal syphilis screening program for pregnant women and found reductions in rates of congenital syphilis. Two studies on screening accuracy for syphilis reported false-positive rates of less than $1 \%$. One study that used a large insurance claims database reported an incidence of anaphylaxis after oral penicillin of 0.1 per 10000 dispensings. In a study from Hungary, oral penicillin in pregnancy was not associated with orofacial clefts.

Limitations: This was a targeted literature search and could have missed small studies on the benefits and harms of screening for syphilis in pregnancy. We did not review evidence on interventions to improve rates of prenatal screening.

Conclusion: New evidence from a study of universal screening supports previous evidence on the effectiveness of screening for syphilis in pregnancy to prevent congenital syphilis. Harms include testing and follow-up for false-positive test results and adverse effects from penicillin treatment.

Ann Intern Med. 2009;150:710-716.

www.annals.org

For author affiliations, see end of text.
S yphilis is caused by the spirochete Treponema pallidum and manifests as a systemic infectious process. Syphilis may be transmitted vertically, usually through the placenta; the risk for fetal infection increases with gestational age. Vertical transmission may also occasionally occur during delivery if maternal genital lesions are present (1). The consequences of fetal syphilis include prematurity, low birthweight, nonimmune hydrops, and intrauterine death (2).

Associated conditions and risk factors for syphilis among reproductive-age women in the United States include substance abuse, limited access to health care, poverty, African-American ethnicity, and lack of regular prenatal care $(3,4)$. In addition, rates of primary and secondary syphilis are highest among individuals 25 to 29

See also:

\section{Print}

Related article. . . . . . . . . . . . . . . . . . 705

Summary for Patients. . . . . . . . . . . . . . . . . I-40

Web-Only

Conversion of graphics into slides

Downloadable recommendation summary years of age (5). Rates of syphilis among women have increased since 2004. Between 2005 and 2006, rates increased among women by $11.1 \%$ (from 0.9 to 1.0 cases per 100000 persons) (5). In the United States, the number of babies born with syphilis has consistently decreased, from 4410 cases in 1991 to 353 cases (8.8 cases per 100000 live births) in 2004 (4). However, the rate of congenital syphilis increased between 2005 and 2006 by $3.7 \%$ (from 8.2 to 8.5 cases per 100000 live births) (3). Although the overall rate of congenital syphilis has decreased significantly since the onset of the syphilis elimination plan in 1996, this recent increase is cause for concern, given that congenital syphilis is preventable (5).

In 2004, the U.S. Preventive Services Task Force (USPSTF) strongly recommended that clinicians screen all pregnant women for syphilis infection $(6,7)$. Our purpose is to provide information for the USPSTF to update, and reaffirm, its 2004 recommendation. As a basis for this reaffirmation, the USPSTF asked us to do a targeted literature search to find new and substantial evidence on the benefits and harms of screening for syphilis in pregnancy and the harms of treatment with penicillin. Topics that undergo a reaffirmation update have already undergone a previous systematic USPSTF review; in addition, these topics represent standards of care that are well established, 
evidence-based, and in current medical practice (8). The USPSTF performs reaffirmation updates for topics that remain a Task Force priority, that are within the scope of review of the USPSTF, and for which the USPSTF has a convincing reason to keep the recommendation current (8).

The USPSTF requested that this reaffirmation update address the following primary key questions:

1. Does screening for syphilis in pregnancy reduce the prevalence of congenital syphilis in neonates?

2. Are there harms of screening for syphilis or harms of treatment with penicillin in pregnancy to women or neonates?

The USPSTF determined that for this update it was not necessary to review the accuracy of screening tests or the effectiveness of treatment in neonates and in pregnant women. The accuracy of rapid plasma reagin (RPR) and Venereal Disease Research Laboratory (VDRL) screening tests and penicillin treatment are well established. The USPSTF is, however, interested in any potential harms of these screening tests, as well as potential harms of penicillin treatment in neonates and pregnant women, as indicated in the key questions above.

\section{Methods}

\section{Data Sources and Searches}

We performed literature searches on the benefits and harms of screening for syphilis infection in pregnant women, as well as the harms of penicillin treatment for this infection in pregnant women and in neonates. We limited our searches to the period of 1 January 2003 through 31 July 2008 and used the search terms penicillin, pregnancy, infant, newborn, fetus, adverse effect, allergic reaction, harm, mass screening, rapid plasma reagin, VDRL antigen, pregnancy complications, Treponema pallidum, and syphilis. We limited our initial searches to English-language articles that were indexed in the PubMed core clinical journal subset (formerly known as the Abridged Index Medicus). We supplemented these searches by reviewing reference lists of important articles and recent reviews and by taking suggestions from experts.

\section{Study Selection}

We selected studies that provided evidence on the benefits of screening for syphilis in pregnancy in the reduction of incidence of congenital syphilis; the harms of screening, specifically focusing on false-positive and false-negative results; and the harms of treatment, primarily allergic reactions and fetal harms. For evidence on benefits, we selected studies that included pregnant women. For evidence on false-positive and false-negative results, we included studies in pregnant and nonpregnant adults who were screened with RPR or VDRL and used treponemal-specific tests as the gold standard. We excluded studies that reported only results for newer rapid tests and did not report results on
RPR and VDRL, which are considered the standard of care in the United States. For evidence on allergic reactions to penicillin, we selected studies that included pregnant and nonpregnant adults. We excluded studies in high-risk or special populations and studies in populations not generalizable to the United States. We determined generalizability of study sample to the United States by consensus of 2 reviewers after discussions with the USPSTF on similarities between the health care system in the study country and that of the United States. Considerations about whether a population would be comparable to a U.S. population include the general health status of the population, the availability of prenatal care, and the availability of trained delivery attendants. We specifically excluded studies in populations with high HIV rates, because these studies are thought not to be generalizable to the United States.

To determine whether prenatal screening reduces the prevalence of congenital syphilis in neonates, we included randomized, controlled trials; meta-analyses; and systematic reviews. In addition, we included large ecologic studies and cohort studies that reported the effect of the implementation of widespread screening programs. We included these types of studies because the original evidence on the effectiveness of syphilis screening in pregnancy was from ecologic studies that showed that rates of congenital syphilis were reduced after widespread screening and treatment. To determine the harms of syphilis screening and penicillin treatment, we included randomized, controlled trials; metaanalyses; systematic reviews; cohort studies; case-control studies; and large case series. We excluded editorials, narrative reviews, case studies, and guideline reports.

At the abstract and full article review stage, 2 reviewers independently evaluated all articles according to predetermined exclusion criteria. Any article selected by at least 1 reviewer at the abstract stage was advanced to the full article stage of the review. We resolved differences of opinion at the full article stage by consensus and involved a third reviewer if necessary.

\section{Data Extraction}

We extracted information from each included study on its design, selection criteria, demographic characteristics, and clinical outcomes.

\section{Quality Appraisal}

We provided narrative descriptions of key methodological deficiencies of included studies that constrain the quality and generalizability of the evidence.

\section{Data Synthesis}

We synthesized the evidence from included studies in a narrative format.

\section{Role of the Funding Source}

The work conducted by the USPSTF is supported by the Agency for Healthcare Research and Quality. This review did not receive separate funding.

19 May $2009 \mid$ Annals of Internal Medicine $\mid$ Volume 150 • Number $10 \mid \mathbf{7 1 1}$ 


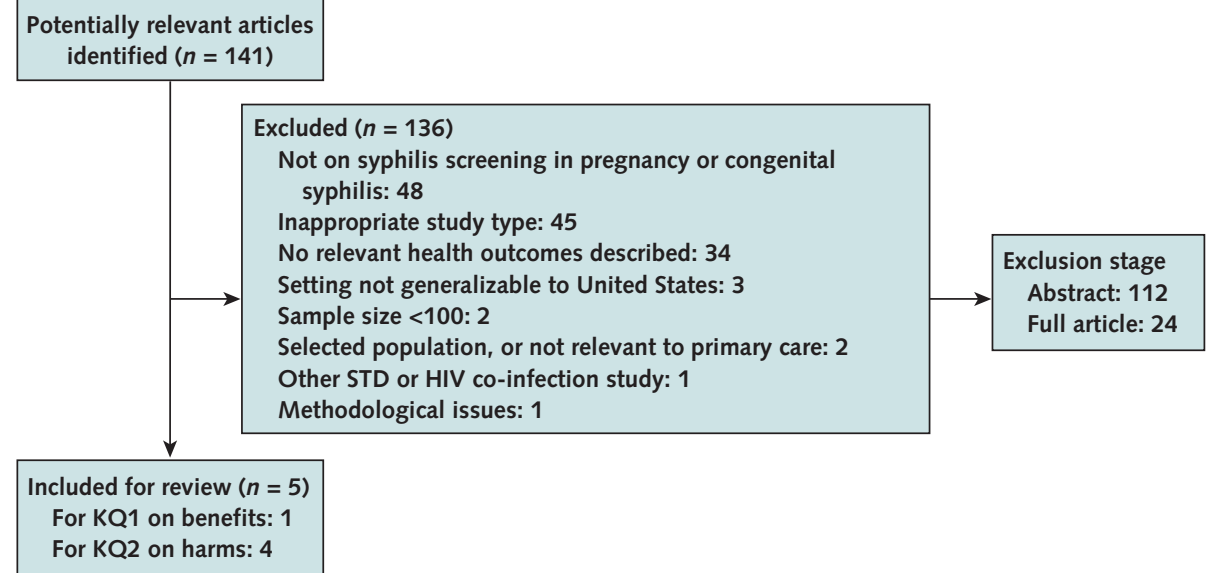

$\mathrm{KQ}=$ key question; STD $=$ sexually transmitted disease.

\section{RESULTS}

We identified 141 potentially relevant studies. We most commonly excluded studies because they were not on syphilis screening or treatment, were not an appropriate study type, or had no information on relevant health outcomes. After we excluded studies on the basis of predetermined criteria (Figure), 5 studies remained (Table) 1 study of the benefits of screening and 4 studies of the harms of screening and treatment.

\section{Key Question 1}

Does screening for syphilis in pregnancy reduce the incidence of congenital syphilis in neonates?

We found 1 study in a non-U.S. population (9) that provided information on the benefits of screening for syphilis in pregnancy and met our inclusion criteria; we did not find any new studies in U.S. populations. The 1 study we found evaluated the effect of implementing a free, routine syphilis screening program for pregnant women in a region of China. A total of 418871 pregnant women who were treated at 61 hospitals in the Shenzhen region of China from 2003 to 2005 were offered free syphilis screening. Ninety-four percent of eligible women were screened. Syphilis was diagnosed in 2019 women over the 3 years of the study, of whom $92 \%$ received timely treatment, $3 \%$ received delayed treatment, $3 \%$ declined treatment, and $2 \%$ were lost to follow-up. Of the 1402 women who had syphilis and chose to continue their pregnancy, $86(6 \%)$ had pregnancies that ended in fetal demise or stillbirth. Ninety-two live-born infants with congenital syphilis were reported in the 3 years of the study. Most $(83 \%)$ of these cases were in babies born to women who received no prenatal care. Eight percent occurred in babies born to women who received late prenatal care, and 5\% occurred in babies born to women who declined treatment. The incidence of congenital syphilis decreased after the implementation of this screening program: At the beginning of the study, the incidence rate was 54 cases per 100000 pregnant women; the rate decreased to 22 cases per 100000 pregnant women after initiation of the program. The study investigators report that loss to follow-up was an important limitation because many migratory women changed contact information frequently. In addition, many pregnant women returned to their hometown to deliver their babies.

\section{Key Question 2}

Are there harms of screening for syphilis or harms of treatment with penicillin in pregnancy to women or neonates?

\section{Harms of Screening}

Potential harms of screening may include opportunity costs to the clinician and patient, including time and resources, and false-positive results, which may lead to stress, labeling, and further work-up. We found limited evidence on these potential harms of screening; the previous review for the USPSTF found no studies that addressed these potential harms. We did not find any studies on the downstream harms of screening. We did find 2 studies on the harms of screening that evaluated the accuracy of the RPR and VDRL syphilis screening tests. Although we did not review the evidence on the accuracy of screening, we include these studies as evidence on the harms of screening because they report information on false-positive and falsenegative results. The first study (10) is a retrospective study that used data from a large database of sera results from more than 300000 patients who were routinely tested for syphilis while hospitalized in Vienna hospitals from 1988 through 1999. The investigators report frequencies of biological false-positive results, defined as the number of patients who have a positive VDRL result and a negative T. pallidum hemagglutination result divided by the total 
number of sera tested. The overall incidence of syphilis in this study (defined as reactive on the $T$. pallidum hemagglutination test) was approximately $1.8 \%$. Biological falsepositive results occurred in $0.26 \%$ of all patients. The rate of biological false-positive results was $0.26 \%$ for women age 21 to 30 years and $0.22 \%$ for women age 31 to 40 years. This study has limited applicability to our review because of the lack of separately reported data on pregnant women. Another limitation is that these patients were all hospitalized and may differ from women who present for routine outpatient prenatal care.

The second study that provided information on the harm of false-positive results (11) evaluated 8892 pregnant women who presented for prenatal care at 4 large urban hospitals in Bolivia between January 2004 and April 2005. Rapid plasma reagin screening results were compared with the T. pallidum particle agglutination assay (the gold standard). Seventy-eight false-positive RPR results were reported, for a rate of $0.91 \%$ (78 [the number of falsepositive results] divided by 8550 [the number of patients with negative $T$. pallidum particle agglutination assay results]); 83 women with a negative RPR had a positive T. pallidum hemagglutination result, which indicates likely previous infection. Three hundred forty-two women had positive T. pallidum particle agglutination assay results.

\section{Harms of Treatment With Penicillin}

In addition to the harms of screening, potential harms may also result from treatment in response to positive screening results. We searched for evidence on harms to the woman and fetus of treatment with penicillin, the accepted treatment for syphilis in pregnancy. We found 1 study (12) that provided information on serious allergic reactions to penicillin. Investigators used a large U.S. insurance claims database of approximately 10 million patients to calculate the frequency of serious allergic reactions within 14 days of receiving penicillin (12). They defined a serious allergic reaction as a claim for services at a hospital or emergency department for an International Classification of Diseases, Ninth Revision, code; Current Procedural Terminology code; or procedural code for anaphylactic shock, unspecified adverse effect of drug, unspecified allergy, cardiopulmonary resuscitation, or adrenaline injection. The investigators reviewed medical records to confirm the diagnosis of anaphylaxis. Penicillin was administered 199862 times during the course of the study; $53 \%$ of those were to women and $35 \%$ to adults 20 to 39 years of age. The incidence of anaphylaxis after penicillin was received was 0.1 per 10000 dispensings; resuscitation, 0.2 per 10000 dispensings; adverse effect of drug, 2.1 per 10000 dispensings; allergy, 2.4 per 10000 dispensings; and any allergic reaction, 4.7 per 10000 dispensings. An important limitation of this study was the reliance on claims data, which may contain miscodings. The investigators reviewed medical records for $83 \%$ of patients with claims for anaphylaxis and resuscitation and con- firmed the diagnosis of anaphylaxis in 3\% of resuscitationcoded claims and 57\% of anaphylaxis-coded claims. The lack of information on incidence in pregnant women further limits the applicability of this study to our review. In addition, this study reports the incidence of allergic reactions after oral penicillin; however, penicillin treatment for syphilis is given parenterally.

We found 1 study that provides information on the harms of penicillin to the fetus during pregnancy. Puhó and colleagues (13) used data from the 1980 to 1996 Hungarian Congenital Abnormality Registry that includes information from mandatory reports by physicians of any congenital abnormalities in Hungary. The investigators identified 1374 cases of isolated orofacial clefts and reviewed medical records and questionnaire results for medication use. The prevalence of isolated orofacial clefts in children born to women who received penamecillin was compared with a control population and a control group with noncleft malformations. Penamecillin is an oral form of penicillin not available in the United States. The investigators found no association between penamecillin and isolated orofacial clefts.

\section{DISCUSSION}

Congenital syphilis is a preventable disease that continues to be an important public health issue in the United States, despite strong evidence of effective treatment and widespread screening. In the United States, $90 \%$ of the states mandated prenatal syphilis testing in 2003 (14). However, many cases in the United States are attributable to late or no prenatal care. In 2002, a total of 451 congenital syphilis cases were reported to the Centers for Disease Control and Prevention; of these, $73.8 \%$ occurred in mothers with inadequate treatment of syphilis before or during pregnancy, including $22.6 \%$ who did not have an adequate serologic response to therapy (15). Moreover, $29 \%$ of mothers of infants with congenital syphilis did not receive prenatal care, and only $36 \%$ of mothers for whom we have information about the timing of their prenatal care began to receive care during their first trimester of pregnancy. Provider nonadherence with guidelines also plays a role in preventable cases of congenital syphilis. A national survey from 1999 to 2000 (15) indicated that $14 \%$ of obstetricians/gynecologists did not routinely screen pregnant women for syphilis, and that many did so only once for women at high risk. Further research should focus on ways to increase the receipt of early prenatal care in women at high risk and increase appropriate adherence to treatment and follow-up.

We found evidence primarily with respect to 1 particular harm of screening, false-positive results. Relationship disruptions resulting from syphilis screening have also been reported. We did not find studies on this but did not search specifically for it. We did find evidence on serious 
Clinical Guidelines Screening for Syphilis Infection in Pregnant Women

Table. New Studies on the Benefits of Screening and Harms of Screening and Treatment for Syphilis in Pregnancy

\begin{tabular}{|c|c|c|}
\hline Study, Year (Reference) & Study Design & Objective \\
\hline Cheng et al, 2007 (9) & Prospective cohort & $\begin{array}{l}\text { To understand the } \\
\text { epidemiology of } \\
\text { syphilis in pregnant } \\
\text { women and evaluate } \\
\text { the effectiveness of a } \\
\text { screening and } \\
\text { intervention program } \\
\text { to control vertical } \\
\text { (mother-to-fetus) } \\
\text { transmission in } \\
\text { Shenzhen, China. }\end{array}$ \\
\hline Johannes et al, 2007 (12) & Retrospective cohort & $\begin{array}{l}\text { To conduct a systematic } \\
\text { study estimating the } \\
\text { drug-specific } \\
\text { incidence of serious } \\
\text { allergic reactions to } \\
\text { antibiotics in a known } \\
\text { population in the } \\
\text { United States. }\end{array}$ \\
\hline
\end{tabular}

Tinajeros et al, 2006 (11) Prospective cohort To evaluate the performance of POC syphilis tests at maternity hospitals in urban Bolivia.

\section{Sample Population and Selection Criteria}

477656 eligible women (>3 mo pregnant and living in Shenzhen, China; $94 \%$ coverage) seeking prenatal care at registered facilities were screened for syphilis between 1 July 2002 and 31 December 2005. Program offered free routine syphilis screening to all pregnant women and covered 61 hospitals in all 6 districts by July 2002 .
Claims and medical records of patients in a large U.S. health insurer database (covering 10 million patients in the Midwest and Southeast) who received antibiotics between 1 July 2000 and 30 June 2004. Received penicillin: all dispensings, 250 598; first dispensing, 199862

8924 pregnant women older than $18 y$ who requested prenatal care, were not tested for syphilis earlier in pregnancy, and consented to participate and have blood drawn. Enrollment at 4 urban maternity hospitals in Bolivia (large, public, with laboratory infrastructure and $3 \%$ syphilis prevalence) during busy morning shift between January 2004 and April 2005.
Puhó et al, 2007 (13)

\section{Case-control}

To evaluate possible
association between
drug treatment
during pregnancy and
isolated OFC in the
offspring.

Geusau et al, 2005 (10)

\section{To determine frequency} of biological falsepositive results by using VDRL as a screening test.
Mandatory notification to HCAR since 1962, including fetal defects since 1984 , with $96 \%$ of OFCs reported. The HCCSCA, 1980-1996, includes HCAR cases with CL/P and PCP and control participants with malformations reported within 3 months after birth or termination.

\section{Sera from 301032 patients at General} Hospital of Vienna from 1988 to 1999 for whom age and sex data were available.
Intervention and Comparison

Screened by using TRUST and confirmed by using TPPA or FTA-ABS. If positive, advised to accept follow-up visits and treatment and to decide whether to continue their pregnancies on the basis of risk evaluation results. Penicillin: 2.4 MU/wk intramuscular $\times 3$; $0.8 \mathrm{MU} / \mathrm{d}$ intramuscular $\times 14$. Erythromycin: $0.5 \mathrm{~g} 4$ times/d $\times$ $14 \mathrm{~d}$. Treatment given at diagnosis or during weeks 30-32. Follow-up for 24 mo after delivery. Infants of treated women screened at birth.

Cohort exposed to antibiotic were followed for $14 \mathrm{~d}$ after receipt for ICD-9 codes consistent with outcomes of allergy, anaphylaxis, or CPR, and aggregated as "any allergic reaction." Events after exposure were compared with each patient's baseline period $183 \mathrm{~d}$ before index date of study drug receipt.

Finger-stick POC test using Abbott Determine Syphilis TP test (Abbott Laboratories, Abbott Park, Illinois). Venous sample for serum RPR on site at hospital and frozen aliquot sent for reference RPR and confirmatory TPPA. Women testing positive by POC or TPPA were treated for syphilis according to WHO guidelines. True-positive and true-negative results were defined by positive RPR and TPPA results at reference laboratory.

Compared treatments or supplements used by at least $0.5 \%$ of pregnant women during critical periods who delivered children with OFC. Population control participants without congenital abnormalities matched 2-3:1 to case patients according to sex, birth week, and district. Captured prospective medical record data, retrospective maternal self-reports, and supplementary data from regional nurses.

Sera samples tested by using TPHA (serial dilutions, considered reactive at $>1: 80$ ) and VDRL (diluted to end point titer, with highest reactive dilution reported). Samples were considered to have biological false-positive results if they were VDRL-positive but TPHAnegative.

$\mathrm{CL} / \mathrm{P}=$ uni- and bilateral cleft lip and cleft lip and palate; $\mathrm{CPR}=$ cardiopulmonary resuscitation; FTA-ABS $=$ fluorescent treponemal antibody absorption; HCAR $=$ Hungarian Congenital Abnormality Registry; HCCSCA = Hungarian Control Surveillance of Congenital Abnormalities; ICD-9 = International Classification of Diseases, Ninth Revision; $\mathrm{OFC}=$ orofacial cleft; $\mathrm{PCP}=$ posterior cleft palate; $\mathrm{POC}=$ point of care; $\mathrm{POR}=$ prevalence odds ratio; $\mathrm{RPR}=$ rapid plasma reagin; $\mathrm{TPHA}=$ Treponema pallidum hemagglutination test; TPPA $=$ Treponema pallidum particle agglutination test; TRUST $=$ toluidine red unheated serum test; VDRL $=$ Venereal Disease Research Laboratory test; $\mathrm{WHO}=$ World Health Organization. 
Screening for Syphilis Infection in Pregnant Women $\mid$ CLiniCA L Guidelines

Table-Continued

Results

$2208(0.5 \%)$ tested positive; syphilis diagnosed in 2019. 1112 (79\%) were followed until childbirth. 92 cases of congenital syphilis in 3 years. 22 cases per 100000 pregnant women over 3 years, lower than the rate of 53.6 before the start of the screening program. $4.6 \%$ rate of congenital syphilis in women who tested positive. Success rate of intervention, $99 \%$ (1752 of 1768$)$

2 anaphylactic reactions identified (0.1 per 100000 dispensings [95\% Cl, 0.0-0.3]). 114 allergic reactions after all dispensings (4.6 per 100000 dispensings [Cl, 3.8-5.4]). 93 allergic reactions after first dispensing (4.7 per 100000 dispensings $[\mathrm{Cl}$ 3.8-5.7]). Previous reports of 1-5 anaphylactic reactions per 100000 treatments.
Additional Information

1855 (92\%) opted for treatment; 63 delayed treatment; 54 declined; and no data were available for 47 patients. 296 patients had miscarriage, stillbirth, or ectopic pregnancy; 274 chose termination. 1402 patients chose to continue their pregnancy, of whom 86 had adverse outcomes; 204 were lost to follow-up. 76 babies with congenital syphilis were born to women not screened by the program: 4 to women in whom treatment failed, 5 to women who declined treatment, and 7 to women who received late treatments. Success rate defined as number of infants without congenital syphilis over number of mothers who tested positive and received intervention.

Other antibiotics studied: moxifloxacin, levofloxacin, gaifloxacin, ciprofloxacin, and cephalosporins. Unlike previous estimates that used spontaneous reports, denominators of incidence rates in this study reflect the population from which the cases arose.
Summary

Screening pregnant women is effective in enabling treatment and efficient in preventing congenital syphilis. Women with positive screening results are more likely to be young, migratory, and of lower socioeconomic status. Mothers who never had prenatal examinations accounted for the highest percentage $(63 \%)$ of babies with congenital syphilis, followed by cases of childbirth outside hospitals (20\%), and treatment late in pregnancy $(8 \%)$
342 confirmed cases among 8892 women who completed screening. Prevalence, $3.84 \%(\mathrm{Cl}$ $3.5 \%-4.3 \%)$. POC sensitivity, $91.8 \%(\mathrm{Cl}$, $88.4 \%-94.5 \%)$. Hospital RPR sensitivity, $75.7 \%(\mathrm{Cl}$ $70.8 \%-80.2 \%)$. POC prevalence, 442 (5\%) cases (Cl, 4.8\%-5.2\%). Specificity: POC, 98.5\%; hospital RPR, 99.0\%. Positive predictive value: POC, $71 \%$; hospital RPR, 77\%. Negative predictive value: POC, 99.7\%; hospital RPR, 99\%.
Bolivian surveillance system estimates that only half of women return for RPR results, so only $38 \%$ of women who test positive receive treatment. Assuming a 30\% rate of congenital syphilis, POC testing averted 61 cases (54\% of 114 cases). 32 cited cases of penicillin anaphylaxis per 100000 exposed. Participants given a baby blanket.
The incidence of any allergic reaction after firs eligible or all dispensings was lowest for penicillin, compared with fluoroquinolones and cephalosporins. Lower incidence of penicillin anaphylaxis was attributed to oral route, preselection if penicillin allergy was identified, mislabeling as allergic events, and inclusion of hospital cases only.
More sensitive and equally specific results from POC vs. hospital RPR, with $128(1.4 \%)$ falsepositive results and 28 of 8892 false-negative results. POC increased uptake and ensured treatment delivery in a low-resource setting. Mentions risks of misdiagnosis, including adverse penicillin reaction, stigma, and social consequences, including intimate partner violence.
Penamecillin POR: $1.1(\mathrm{Cl}, 0.8-1.5)$ for $\mathrm{CL} / \mathrm{P}$ compared with population control participants, $1.0(\mathrm{Cl}, 0.7-1.3)$ for CL/P compared with control participants with malformations, $1.2(\mathrm{Cl}, 0.7-1.9)$ for PCP compared with population control participants, $1.1(\mathrm{Cl}, 0.7-1.8)$ for PCP compared with control participants with malformations. Phenoxymethyl-penicillin POR: 1.8 (Cl, 0.6-5.8) for PCP compared with population control participants, $1.6(\mathrm{Cl}, 0.6-4.3)$ for PCP compared with control participants with malformations. Amoxicillin POR: 15.9 (Cl, 4.9-51.2) for CL/P compared with population control participants, $5.4(\mathrm{Cl}, 1.9-15.4)$ for CL/P compared with control participants with malformations.

5320 TPHA-positive $(1.77 \%)$, of which 3257 were VDRL-negative (61.2\%). Biological false-positive reaction detected in $736(0.26 \%$ of all sera). Frequency was higher in women than men, except for men age 31-40 y (because of higher rate of HIV-infected men)
Critical period is 7 to 9 weeks for CL/P and 10 to 14 weeks for PCP. OFC prevalence, 1 case per 750 births. Prenatal care is mandatory in Hungary (nearly 100\%), with an average of 7 prenatal visits. Potential confounders identified separately for 5 medication groups.
This study suggests that drugs-including penicillin-have a limited role in causing both $\mathrm{CL} / \mathrm{P}$ and $\mathrm{PCP}$, confirms the teratogenic effect of certain anticonvulsants, and suggests a possible association between OFC and either the penicillin-derivative amoxicillin or oxprenolol.
VDRL results at dilution 1:4 were not reported for TPHA-negative sera, thus 1451 of 2799 sera could not be directly compared with regard to true or biological false-positive VDRL results.
Agreement with previous studies regarding syphilis prevalence in Austria and more frequent biological false-positive results in women (possibly due to autoimmune comorbid conditions), HIV-positive patients (who have a 10-fold higher rate of biological false-positive results), and people older than $60 \mathrm{y}$. 


\section{Clinical Guidelines $\mid$ Screening for Syphilis Infection in Pregnant Women}

reactions to penicillin; however, these types of reactions seem to be infrequent.

In summary, we found new evidence that supports previous evidence of the effectiveness of screening for syphilis in pregnancy to prevent congenital syphilis. The effectiveness of screening depends on early receipt of prenatal care and appropriate treatment and follow-up of positive test results. We found new evidence of a slight risk for harm from false-positive test results and a rare risk for serious allergic reactions to penicillin.

From the U.S. Preventive Services Task Force, Agency for Healthcare Research and Quality, Rockville, Maryland.

Potential Financial Conflicts of Interest: None disclosed.

Requests for Single Reprints: Reprints are available from the Agency for Healthcare Research and Quality Web site (www.preventiveservices .ahrq.gov)

Current author addresses are available at www.annals.org.

\section{References}

1. Majeroni BA, Ukkadam S. Screening and treatment for sexually transmitted infections in pregnancy. Am Fam Physician. 2007;76:265-70. [PMID: 17695573]

2. Chakraborty R, Luck S. Syphilis is on the increase: the implications for child health. Arch Dis Child. 2008;93:105-9. [PMID: 18208988]

3. Trends in Reportable Sexually Transmitted Diseases in the United States, 2006: National Surveillance Data for Chlamydia, Gonorrhea, and Syphilis. Atlanta: Centers for Disease Control and Prevention; 2007. Accessed at www.cdc. gov/std/stats06/pdf/trends2006.pdf on 8 April 2009.

4. Walker GJ, Walker DG. Congenital syphilis: a continuing but neglected problem. Semin Fetal Neonatal Med. 2007;12:198-206. [PMID: 17336171]
5. Surveillance 2006: National Profile: Syphilis. Atlanta: Centers for Disease Control and Prevention; 2007. Accessed at www.cdc.gov/std/stats06/syphilis.htm on 8 April 2009.

6. Calonge N; U.S. Preventive Services Task Force. Screening for syphilis infection: recommendation statement. Ann Fam Med. 2004;2:362-5. [PMID: 15335137]

7. Nelson HD, Glass N, Huffman L, Villemyer K; Hamilton A, Frame P, et al. Screening for Syphilis: Brief Update. Rockville, MD: Agency for Healthcare Research and Quality; 2004. Accessed at www.ahrq.gov/clinic/3rduspstf/syphilis /syphilup.htm on 8 April 2009.

8. U.S. Preventive Services Task Force. U.S. Preventive Services Task Force Procedure Manual. AHRQ Publication No. 08-05118-EF. Rockville, MD: Agency for Healthcare Research and Quality; 2008. Accessed at www.ahrq.gov /clinic/uspstf08/methods/procmanual.pdf on 8 April 2009.

9. Cheng JQ, Zhou H, Hong FC, Zhang D, Zhang YJ, Pan P, et al. Syphilis screening and intervention in 500,000 pregnant women in Shenzhen, the People's Republic of China. Sex Transm Infect. 2007;83:347-50. [PMID: 17693449]

10. Geusau A, Kittler H, Hein U, Dangl-Erlach E, Stingl G, Tschachler E. Biological false-positive tests comprise a high proportion of Venereal Disease Research Laboratory reactions in an analysis of 300,000 sera. Int J STD AIDS. 2005;16:722-6. [PMID: 16303064]

11. Tinajeros F, Grossman D, Richmond K, Steele M, Garcia SG, Zegarra L, et al. Diagnostic accuracy of a point-of-care syphilis test when used among pregnant women in Bolivia. Sex Transm Infect. 2006;82 Suppl 5:v17-21. [PMID: 17121762]

12. Johannes CB, Ziyadeh N, Seeger JD, Tucker E, Reiter C, Faich G. Incidence of allergic reactions associated with antibacterial use in a large, managed care organisation. Drug Saf. 2007;30:705-13. [PMID: 17696583]

13. Puhó EH, Szunyogh M, Métneki J, Czeizel AE. Drug treatment during pregnancy and isolated orofacial clefts in Hungary. Cleft Palate Craniofac J. 2007;44:194-202. [PMID: 17328645]

14. Hollier LM, Hill J, Sheffield JS, Wendel GD Jr. State laws regarding prenatal syphilis screening in the United States. Am J Obstet Gynecol. 2003;189: 1178-83. [PMID: 14586375]

15. Centers for Disease Control and Prevention (CDC). Congenital syphilisUnited States, 2002. MMWR Morb Mortal Wkly Rep. 2004;53:716-9. [PMID: 15306757] 


\section{Annals of Internal Medicine}

Current Author Addresses: Drs. Wolff and Miller: Center for Primary Care, Prevention, and Clinical Partnerships, Agency for Healthcare Research and Quality, 540 Gaither Road, Rockville, MD 20850.

Dr. Shelton: Johns Hopkins Bloomberg School of Public Health, 615 North Wolfe Street, Room WB602, Baltimore, MD 21205-1996.
Dr. Sessions: Uniformed Services University of the Health Sciences, PMB Department, Room A-1040A, 4301 Jones Bridge Road, Bethesda, MD 20814. 